\title{
Labyrinthe
}

$7 \mid 2000$

Numéro 7

\section{L'identité légale des Juifs sous Vichy}

La contribution des juges

\section{Philippe Fabre}

\section{CpenEdition}

\section{Journals}

Édition électronique

URL : http://journals.openedition.org/labyrinthe/501

DOI : 10.4000/labyrinthe.501

ISSN : 1950-6031

Éditeur

Hermann

\section{Édition imprimée}

Date de publication : 30 octobre 2000

Pagination : 23-41

\section{Référence électronique}

Philippe Fabre, «L'identité légale des Juifs sous Vichy », Labyrinthe [En ligne], 7 | 2000, mis en ligne le 28 mars 2005, consulté le 30 avril 2019. URL : http://journals.openedition.org/labyrinthe/501 ; DOI : 10.4000/labyrinthe.501

Ce document a été généré automatiquement le 30 avril 2019.

Propriété intellectuelle 


\title{
L'identité légale des Juifs sous Vichy
}

\author{
La contribution des juges
}

Philippe Fabre

Cet article constitue la reprise synthétique de certains développements d'un mémoire de DEA en droit public sous la direction de Gérard Conac et Herbert Maisl, soutenu en 1998 à l'Université Paris I-Panthéon-Sorbonne, "Le Conseil d'État et le régime de Vichy : le contentieux de l'antisémitisme ", édité cet automne aux Publications de la Sorbonne dans la collection «De Rebus Publicis » qu'il inaugure. S'en

rapportant à cet ouvrage, on a ici limité des

indications bibliographiques facilement multipliables eu égard au nombre de travaux intéressant la période ; presque toutes celles qui ont été retenues sont par conséquent à entendre entre autres. «Si le juif n'existait pas, l'antisémite

l'inventerait. » J.-P. Sartre

«Emprisonner les hommes, c'est peu ; les tuer, ce n'est pas grand chose ; les juger avec le motif, c'est bien. »

J. Barthélémy (garde des Sceaux, 1941-1943)

1 Le 3 octobre 1940, le régime de Vichy adopte la première « loi portant statut des juifs ", œuvre du garde des Sceaux Raphaël Alibert ; elle est publiée au Journal officiel le $18^{1}$. Trois mois à peine après le "suicide » de la République - le vote du 10 juillet, les pleins pouvoirs accordés à Pétain -, l'« État français » concrétise les projets d'une décennie d'antisémitisme, et le ressentiment de plusieurs siècles ${ }^{2}$. Une communauté entière, interdite de la plupart des fonctions publiques ou privées de direction ou d'influence, se 
trouve mise au banc de la société, acte délibéré du gouvernement de la zone libre, indépendant de toute pression réelle de l'Allemagne hitlérienne ${ }^{3}$.

2 «La haine est une occasion de production juridique ${ }^{4}$ » elle l'est aussi, et ce faisant même, d'identité : l'application de ces dispositions requiert nécessairement une définition de la nouvelle catégorie de droit ainsi créée, le «Juif ». Aussi l'article $1^{\mathrm{er}}$ de la loi répute-t-il juive «toute personne issue de trois grands-parents de race juive ou de deux grandsparents de la même race, si son conjoint lui-même est juif ». Singulièrement flou en raison de la base idéologique raciale sur laquelle il s'appuie, ce critère se montre toutefois plus sévère que celui que les Allemands eux-mêmes viennent d'instituer dans la France occupée 5 .

3 Au début de l'année 1941, alors que les pressions nazies s'affirment parallèlement à la volonté du régime de consolider les mesures hâtives de l'automne 1940, la politique antijuive de Vichy subit une inflexion majeure, double mouvement d'intensification. Renforcement des moyens matériels, il donne d'abord naissance, le 29 mars, au « Commissariat général aux Questions juives » (C.G.Q.J.), cheville ouvrière de toutes les mesures antisémites de l'État français jusqu'à sa chute ${ }^{6}$. Durcissement de ces mesures, il conduit dès le 2 juin, sous l'autorité du nouveau commissaire général Xavier Vallat, à la réforme du statut des Juifs.

4 L'« amélioration » du dispositif ${ }^{7}$ passe par la précision de sa cible : avant d'étendre les interdits professionnels à une série d'activités marchandes ou financières, ce « statut Vallat » fixe un nouveau critère de la judéité, plus élaboré que ne l'était le « critère Alibert ", reposant sur l'appartenance religieuse tout en conservant un fondement racial. De la sorte, désormais, « est regardé comme juif : $1^{\circ}$ Celui ou celle, appartenant ou non à une confession quelconque, qui est issu d'au moins trois grands-parents de race juive, ou de deux seulement si son conjoint est lui-même issu de deux grands-parents de race juive. Est regardé comme étant de race juive le grand-parent ayant appartenu à la religion juive ; $2^{\circ}$ Celui ou celle qui appartient à la religion juive, ou y appartenait le 25 juin 1940, et qui est issu d'au moins deux grands-parents de race juive. La non-appartenance à la religion juive est établie par la preuve de l'adhésion à l'une des autres confessions reconnues par l'État avant la loi du 9 décembre $1905^{8}$.»

5 On sait l'enjeu de cette définition dans la France de Vichy, en termes juridiques, plus encore humains. Non seulement la qualification acquise au regard des dispositions précitées scelle une exclusion d'à peu près tous les emplois salariés non manuels, tandis que progressivement des numerus clausus sont arrêtés pour limiter l'accès aux professions libérales 9 , elle impose aussi une obligation de recensement (issue de la loi du 2 juin 1941 accompagnant le statut du même jour), et le cas échéant engage les spoliations destinées à assurer l'« aryanisation économique » du pays (organisée par la loi du 21 juillet 1941 10). Les "Juifs selon la loi ", ainsi devenus facilement repérables et considérablement fragilisés, seront avec une efficacité accrue internés - la loi l'autorise depuis octobre 1940, sur simple décision préfectorale ${ }^{11}$-, traqués et déportés dès lors qu'à l'été 1942 les autorités françaises accepteront de collaborer au programme génocidaire de la « Solution finale $»^{12}$.

6 Dans ce contexte aujourd'hui bien connu, domaine privilégié, voire surinvesti, de la recherche en histoire contemporaine ${ }^{13}$, et actif foyer, sinon plaie vive, de la mémoire collective $^{14}$, le rôle qu'ont tenu les juges - de toutes juridictions : civile lato sensu, administrative, répressive - est moins clair dans les esprits. Sans nul doute est-ce le fait 
d'un intérêt historiographique plus tardif pour le produit de l'activité de ces dernières durant la guerre ${ }^{15}$. Lui-même paraît devoir s'expliquer par la conjonction de deux phénomènes qui mutuellement se confortent : d'une part, les fortes réticences que semblent avoir longtemps éprouvées des juristes, universitaires ou membres des institutions judiciaires, qui en France assurent traditionnellement la fonction d'historiographes principaux de leur propre corporation ; d'autre part, le faible attachement que les «non juristes », et partant les représentants de la faculté d'histoire majoritairement, ont l'habitude de témoigner aux phénomènes du droit.

7 Pourtant, face à un dispositif dont une particularité consistait dans sa formulation quasi exclusivement juridique, l'association des tribunaux aux visées antisémites de l'État français s'avérait inéluctable, les mesures individuelles d'application ne pouvant manquer de déboucher sur une contestation dont la résolution passait par la mise en œuvre juridictionnelle de ce corpus juris, fût-il " monstrueux ${ }^{16}$ ». La contrainte politique qui pesait sur les juges n'était pas négligeable et périlleux le refus d'appliquer les textes antijuifs sous un gouvernement aussi omnipotent qu'autoritaire $-\mathrm{y}$ compris dans sa gestion de l'appareil judiciaire ${ }^{17}$ - et qui avait inscrit ces dispositions dans son plan de redressement général, la " Révolution nationale ». Mais l'obligation relevait d'abord de l'ordre institutionnel, le juge devant se tenir à la loi qu'il a la charge de faire respecter ${ }^{18} \ldots$,., sauf à considérer que les actes dits " lois " du gouvernement de Vichy n'en étaient précisément pas, comme le défendaient au même moment en effet les théoriciens de la France libre ralliée à De Gaulle ${ }^{19}$ - position délicate pour les juridictions de la France occupée, et dont elles se gardèrent, à en croire les revues spécialisées de l'époque ${ }^{20}$, sans exception.

8 À cet égard, le cas du juge administratif - c'est-à-dire alors, du moins pour l'essentiel, du Conseil d'État statuant au contentieux ${ }^{21}$ - se révèle exemplaire à un double titre. En premier lieu, parce qu'à l'instar de ses homologues judiciaires il ne mit pas en cause la validité des lois de Vichy, en particulier des lois antijuives qu'il appliqua comme n'importe quelles autres ${ }^{22}$. En second lieu, parce que, saisi au printemps 1944 d'une requête qui sur un terrain fiscal tendait à contester cette validité, il eut même l'occasion de réaffirmer explicitement, en faveur de ces lois non démocratiques, une jurisprudence traditionnelle consacrée en temps de République, selon laquelle il ne peut s'ériger en censeur de la loi $^{23}$. Il est vrai que, par ailleurs conseiller du gouvernement, le Conseil d'État, en tant que juridiction, à défaut de réellement subir, pouvait ressentir une contrainte politique plus vive que la majeure partie des tribunaux judiciaires, en organisation dispersée $e^{24}$.

9 Et c'est ainsi que, réduites par nominalisme juridique à appliquer les critères des statuts antisémites, celles des juridictions qui se reconnurent compétentes en la matière ne purent que contribuer à préciser les contours de cette fantasmagorie millénaire, que le droit vichyssois, à l'égal puis à la remorque du droit national-socialiste, venait d'officialiser : le « Juif».

10 Toutes choses égales par ailleurs, c'est potentiellement l'ensemble des juridictions de la France de Vichy qui était voué à examiner la qualité de " Juif » au regard des statuts de 1940 puis de 1941. Dès lors, en effet, que chacune - administrative, civile, répressive - se voyait saisie de situations qui ne constituaient que les effets de la reconnaissance de cette qualité, ces contentieux ne pouvaient trouver d'issue que par la vérification de l'exactitude d'une telle qualification. 
11 Certes, factuellement, les affaires relatives au droit antisémite du régime susceptibles d'être soumises à un juge différaient très sensiblement de nature. La juridiction civile, traditionnellement compétente pour connaître des contentieux du travail, de la famille et de la propriété privée, réglerait ainsi, notamment, des litiges survenus dans la mise en œuvre des interdictions professionnelles qui astreignaient les Juifs dans le secteur privé, des différends nés des séparations de biens (prévues par la loi d'aryanisation de 1941) entre époux jusque-là mariés sous le régime de l'indivision ou de la communauté, dont l'un devait être regardé comme juif au regard des critères légaux, et dont la part des biens devait donc être placée sous séquestre, ou des conflits issus de mesures d'administration provisoire liées à cette aryanisation ${ }^{25}$. Devant les tribunaux répressifs, la plupart des dispositions antisémites se trouvant assorties de sanctions pénales, l'ensemble des stigmatisations juridiques infligées aux Juifs par Vichy - ségrégation professionnelle, spoliations, recensement obligatoire - pouvait faire, en cas de manquement, l'objet de poursuites diligentées par le ministère public $^{26}$. Le juge administratif, enfin, conformément à des règles habituelles de répartition du contentieux, eut l'occasion de se prononcer sur la légalité d'exclusions de fonctionnaires prononcées en raison de leur judéité, de décisions d'aryanisation qui s'analysaient en manifestations de prérogatives de puissance publique ${ }^{27}$, de diverses mesures de police administrative touchant les Juifs ${ }^{28}$.

12 Mais chacune de ces juridictions, à s'en tenir là, aurait également pu admettre sa compétence à apprécier la judéité en fonction des critères légaux, ou la " non-judéité ", dès l'instant que de cette qualification dépendait la résolution de cas par ailleurs valablement portés devant elle. C'eût été répondre vite, toutefois, à la question procédurale nouvelle que, faute de disposition expresse des statuts, posait implicitement l'instauration d'une définition juridique des Juifs : en cas de contestation de cette qualification, quel était le juge compétent ? La réponse qu'empiriquement dut y apporter la jurisprudence n'alla pas sans quelque confusion.

Ordinairement juges de l'état des personnes, les tribunaux civils, dès les premières affaires portées devant eux, se reconnurent compétents sans difficulté apparente à apprécier une judéité désormais légalement définie ${ }^{29}$. Au printemps 1942, rendant ses premiers arrêts sur le sujet, le Conseil d'État s'estima à son tour compétent ${ }^{30}$. La solution, d'ailleurs implicite, alors qu'elle mettait le juge administratif en concurrence avec son homologue civil, n'allait pas de soi. Elle obligea la doctrine à en chercher le fondement ; ces lignes témoignent de son effort de compréhension : "Comme rien ne permet de penser que, ce faisant, le Conseil d'État ait entendu rompre avec une tradition jurisprudentielle fermement établie, qui réserve aux tribunaux civils la connaissance des questions d'état et de capacité civile, il y a lieu d'admettre qu[']il n'a pas estimé que la législation sur les juifs ait fait apparaître un état des personnes nouveau, distinct de ceux antérieurement reconnus. Il a sans doute pensé que les textes intervenus dans cette matière n'ont pas eu pour effet de faire déchoir les juifs de leur état de citoyens français et se sont bornés à les frapper d'incapacité et de sujétions qui ne suffisent pas à constituer un état des personnes nouveau ${ }^{31}$.»

14 Néanmoins, le caractère peu satisfaisant pour la rationalité juridique de cette " cocompétence $^{32}$ » des deux ordres de juridiction sur un même problème conduisit le Conseil d'État à se poser rétrospectivement la question de son opportunité, ou plus exactement Maurice Lagrange, commissaire du gouvernement, à en saisir l'occasion en avril $1943{ }^{33}$. Comme un commentateur le rapporta, auquel il faut s'en remettre faute de publication de ses conclusions, « l'éminent commissaire [...] a soutenu cette thèse que la loi du 2 juin 
1941, assortie des autres lois et règlements concernant les juifs, constitue, par l'ampleur des mesures et des incapacités qu'elle prévoit, une loi relative à l'état des personnes. Il a montré que telle était l'opinion de plusieurs juridictions judiciaires [...]. Il a fait valoir enfin les graves inconvénients qui résulteraient de contradictions de jugements entre les deux ordres ${ }^{34}$. » Et Lagrange - illustrant la fidélité du juge administratif à la méthode exégétique de recherche de la volonté du « législateur » - « pensait, d'autre part, trouver une confirmation de sa thèse dans cette circonstance que [la loi] parle expressément de l'état des juifs ${ }^{35} »$. Au terme du raisonnement, il proposait au Conseil d'État de surseoir à statuer pour saisir le juge civil d'une question préjudicielle destinée à établir l'appartenance ou la non-appartenance du requérant à la catégorie légale des " Juifs ». Mais la Haute juridiction ne suivit pas son commissaire et, maintenant la solution acquise en avril 1942 - peut-être même la confortant par une construction a posteriori dans la mesure où l'intervention de Maurice Lagrange laisse penser qu'à l'origine le Conseil ne s'était pas réellement soucié de la question -, parut donc admettre définitivement que les lois raciales édictées par Vichy s'apparentaient à une législation, non d'état des personnes, mais bien de police.

Les juges répressifs, de leur côté, semblent avoir déployé, en majorité, et dès le début de la période, une habile politique jurisprudentielle de défection. Celle-ci consistait avant tout dans la mise en avant de leur compétence strictement pénale ; la prise en compte de cette spécialisation institutionnelle dans l'organisation judiciaire les conduisait en effet à estimer que la question de l'identification d'un individu comme " Juif » au regard des statuts, en tant qu'elle soulevait un problème d'état des personnes, concernait au principal les tribunaux civils, bien qu'un juge répressif puisse en juger par exception lorsque la résolution même de l'affaire criminelle portée devant lui le réclamait, et pour autant qu'elle ne soulevait pas de difficulté sérieuse ${ }^{36}$. Cette position supposait que les juges pénaux se gardent de faire de la judéité un élément constitutif de l'infraction liée au droit antisémite, c'est-à-dire qu'ils évitent, malgré un contexte qui s'y prêtait fortement, de consacrer cette qualité comme illicite par elle-même ${ }^{37}$ - les statuts de 1940-1941 n'allaient pas tout à fait jusque-là, qui « seulement » $\mathrm{y}$ attachaient certaines conséquences juridiques.

On peut enfin remarquer l'affirmation d'une exclusivité de compétence pour apprécier la judéité dont certains juges civils parurent donner le signe à partir de 1944, et singulièrement au tout début de cette année un jugement Consorts Bass du Tribunal civil de la Seine ${ }^{38}$. Celui-ci énonça " qu'en l'absence de dispositions spéciales attribuant compétence exclusive aux tribunaux administratifs pour connaître des demandes tendant à la détermination de la race, ces demandes doivent être portées devant les tribunaux civils compétents ", « tribunaux de droit commun » - et, au terme d'une argumentation très détaillée afin de répondre à chacun des nombreux moyens soulevés, rejeta en conséquence le refus de compétence qu'avait opposé à l'instance le préfet de police, lequel estimait que seul le juge administratif pouvait être valablement saisi de la question 39. La démonstration n'était-elle liée qu'au souci de respecter les règles classiques du partage des compétences entre ordres, ou faut-il y voir aussi l'intérêt pris par les juges à la garantie des droits des Juifs, ces derniers pouvant en effet gagner à la compétence judiciaire, sinon dans l'interprétation du critère de la judéité, du moins dans l'administration des moyens de preuve contraire?

De la jurisprudence globalement considérée relative à l'appréciation de la judéité au regard des critères légaux, trois traits caractéristiques se détachent. Le principal est que 
l'ensemble des juridictions - le Conseil d'État comme les tribunaux civils, mais aussi ceux des juges répressifs qui, par voie d'exception, se reconnurent compétents à raison de tel ou tel cas -, s'en tinrent souvent à la simple exégèse du texte des statuts, reconnaissant " juifs » les intéressés dès lors que les éléments requis se trouvaient réunis, ne déclarant la " non-judéité » que lorsqu'ils faisaient effectivement défaut. Ce que décidant, dans un sens ou dans l'autre, les jugements se bornaient à décrire la plus classique figure de subsomption juridique des faits sous la loi $^{40}$. Il s'agit en second lieu des investigations généalogiques complexes que durent réaliser les juges afin d'établir la filiation des justiciables ; certaines rédactions " témoignent bien des imbroglios et des situations kafkaïens auxquels conduisait l'ahurissante législation antisémite de Vichy ${ }^{41}$ ». Enfin, on observe d'une manière générale que les juges se sont attachés à réaliser des interprétations strictes, traitant comme normal un texte qui était d'exception. Il est vrai que le statut de 1941 était suffisamment précis pour qu'en la matière l'initiative prétorienne demeure limitée - interpretatio cessat in claris. Aussi le reste ne fut-il qu'affaire de preuve, objet d'une activité jurisprudentielle plus créatrice, et parfois moins honorable.

Dans la France des années 1940-1944, les enjeux qui s'attachaient à la preuve de la « nonjudéité » se confondaient, en miroir inversé, avec ceux que revêtait la qualification de " Juif ». Les aménagements que les juges réalisèrent de son régime, souvent déterminant quant au sort - à tout le moins juridique - des justiciables, furent loin d'être univoques ${ }^{42}$

Les juridictions pénales paraissent avoir développé la jurisprudence la plus favorable aux victimes de l'État vichyste. Statuant à titre exceptionnel sur la question, eu égard à la présomption d'innocence bénéficiant au prévenu, la plupart d'entre elles semblent avoir mis la preuve de la judéité, qui justifiait les infractions poursuivies, à la charge du seul ministère public $^{43}$. La chambre criminelle de la Cour de cassation devait même en consacrer le principe en janvier 1943, dans un arrêt qui précisa en outre que la preuve était libre et pouvait s'administrer par tous moyens ${ }^{44}$. La solution, en l'espèce, ne profita certes pas à l'inculpé, contre lequel la Cour exposa que les juges d'appel, suivant la liberté des moyens probatoires qu'elle reconnaissait et le « pouvoir qui leur appartient de puiser les éléments de leur conviction dans tous les éléments de la cause ", avaient pu retenir l'aveu de sa filiation et de sa confession juives ; l'arrêt, néanmoins, s'en remettant ainsi à l'appréciation souveraine des juges, marquait aussi de manière implicite un refus de suivre le conseiller rapporteur sur l'affaire, Marcel Nast : celui-ci, croyant devoir repérer dans le statut de 1941 une "interversion du fardeau de la preuve ", avait proposé d'instaurer une présomption d'appartenance à la religion juive qui aurait pesé sur toute personne ayant deux grands-parents juifs seulement. Au printemps 1942, la Cour d'appel d'Aix, dans les mêmes circonstances, avait déjà refusé de déclarer la judéité d'un prévenu, estimant qu'on ne pouvait retenir à cet effet, contrairement à ce que le procureur avait allégué, ni l'athéisme de l'individu, ni la consonance de son nom patronymique dans la mesure où " la loi n'attache [...] à la forme ou à l'étymologie de celui-ci [...] aucune présomption de sémitisme ${ }^{45} »$.

Dans le même sens, mais plus virulent dans la forme, ce jugement Dorfmann du Tribunal correctionnel de Toulouse ${ }^{46}$ vaut d'être cité, où les magistrats n'ont pas mâché leurs mots pour refuser de condamner l'individu en cause, dont deux grands-parents étaient juifs en ligne paternelle, et dont en ligne maternelle les grands-parents étaient l'un non juif, l'autre inconnu, lui-même se trouvant appartenir à la religion catholique et marié à une 
catholique : "Attendu que cette solution ne heurte pas seulement le bon sens, mais également les principes du droit pénal et civil ; attendu, au point de vue de la loi pénale, qu'admettre que le grand parent inconnu est présumé de race juive aboutit à une interprétation extensive des textes ; que les lois pénales doivent être interprétées restrictivement, ainsi que l'affirme l'un des principes fondamentaux de notre droit consacré par une jurisprudence plus que séculaire [...] ; qu'une jurisprudence semblable reconnaît comme étant d'interprétation restrictive les lois d'exception [...] ; attendu, au point de vue de la loi civile qui régit les questions d'état dont il s'agit, que le Tribunal n'a même pas à considérer qui a pu être le grand-père paternel de Dorfmann et, partant, à s'enquérir de sa race et de sa religion... ». Ce ton, comme le recours au principe de stricte interprétation des lois pénales, est exemplaire d'une tendance de nombreux juges répressifs - tels du moins qu'on en peut appréhender l'activité à travers les revues juridiques de l'époque - à exploiter au mieux les ressources du droit pour neutraliser les effets de la législation antisémite.

Devant les juridictions civiles, les solutions se révèlent plus contrastées. L'instauration d'une « présomption de judéité » fut également refusée en l'absence de tous les éléments nécessaires à remplir le critère légal, par exemple à l'égard de parents inconnus dont la recherche - et, partant, celle de leur " race » ou confession - se trouvait par ailleurs prohibée par le Code civil ${ }^{47}$. Mais d'une lecture trop stricte des textes pouvaient cependant naître des effets pervers, tel celui de n'autoriser que les moyens de preuve expressément spécifiés; c'est ainsi que, contrairement à d'autres jugements civils ${ }^{48}$, le Tribunal de Rabat, en 1941, retint la judéité d'un individu né de deux grands-parents juifs alors que, athée, celui-ci avait établi n'avoir jamais adhéré à la religion juive, les juges interprétant le statut " Vallat » comme autorisant " l'adhésion à l'Église catholique ou à l'Église réformée de France ou à l'Église de la confession d'Augsbourg " pour « seul mode de preuve positif admis par le législateur pour établir la non-appartenance à la religion juive de l'individu né de deux grands-parents juifs ${ }^{49}$ ». Pis, pour le même tribunal, l'adhésion à la religion catholique ou protestante n'était jugée constitutive d'un moyen de preuve opérant que dans la mesure où elle s'avérait effective : de la sorte, qu'il ait été baptisé n'était pas estimé suffisant à prouver la non-judéité d'un homme circoncis ${ }^{50}$.

La jurisprudence administrative traduit une même ambivalence, d'autant plus prononcée qu'elle émane d'une seule institution. D'un côté, suivant un avis rendu fin 1942 par la formation consulative ad hoc instituée au sein du Conseil d'État - la commission du statut des Juifs ${ }^{51}-$, plusieurs décisions annulèrent des actes administratifs qui, pour appliquer des mesures antisémites, s'étaient exclusivement fondés sur le fait qu'issus de deux grands-parents juifs, les requérants n'avaient pas adhéré à une confession autre que le judaïsme ; le Conseil jugeait qu'il ne résultait « ni du texte » du statut, « ni de l'objet que s'est proposé la loi, que le législateur ait entendu n'admettre que ce moyen de preuve à l'exclusion de tout autre ${ }^{52}$ ». Et lorsque, pour établir leur non-judéité, les requérants se prévalaient d'une adhésion à une autre religion que la religion israélite, le juge administratif adopta des positions relativement neutres. Pour la religion catholique, il prétendit s'en remettre au droit canon ${ }^{53}$. Vis-à-vis de l'Église réformée, les références furent plus vagues, le Conseil d'État, suivant une méthode apparentée au faisceau d'indices concordants, estimant protestant quiconque pouvait être considéré comme tel au regard des règles de la communauté à laquelle il déclarait appartenir ${ }^{54}$. Mais la Haute juridiction fut loin de se montrer toujours aussi bienveillante, et, par ailleurs, admit que les simples présomptions avancées par l'administration pouvaient valablement établir la 
qualité juive d'une personne ; il incombait à cette dernière ou au tiers intéressé d'apporter la preuve contraire, ou du moins son commencement ${ }^{55}$. On aurait pourtant pu, sinon dû, concevoir l'inverse, les textes n'imposant nulle " présomption de judéité »; les magistrats judiciaires, presque tous, semblent avoir épargné aux Juifs cette surenchère d'antisémitisme. En outre, le Conseil d'État se montra assez exigeant dans l'appréciation des éléments de nature à constituer un commencement de preuve. La rigueur des arrêts concernant la matière est exemplifiée par l'argumentation développée dans cette affaire Veuve Maxudian d'avril $1943^{56}$ : «Considérant qu'il est constant que la requérante est issue de deux grands-parents israélites dans la branche maternelle ; que, d'autre part, si elle produit un document provenant de l'église consistoriale de Verdun du culte réformé et duquel il résulte que ses grands-parents paternels Moïse et Sarah Lang ont reçu la bénédiction nuptiale en cette église le 14 mars 1843 , cette pièce n'est pas de nature à établir que lesdits conjoints n'aient appartenu, ni l'un, ni l'autre, à la religion juive, contrairement à la présomption qui ressort de l'ensemble des pièces du dossier ; que, faute d'une telle preuve, la dame Maxudian n'est pas fondée à soutenir qu'elle ne saurait être regardée comme juive au sens de la loi du 2 juin $1941^{57}$... » De même, le Conseil d'État refusa de recevoir la présomption de non-judéité que pouvait paraître apporter le nom patronymique du requérant n'étant pas "de consonance juive ${ }^{58}$ ». En somme, à présomptions égales, celles de l'administration, aux yeux du juge, étaient créditées d'une plus grande force probante que celles des particuliers. Le Conseil ne se montra plus compréhensif à cet égard que dans un arrêt de la toute fin-décembre $1943^{59}$, qui accepta qu'une présomption fragile invoquée par l'administration puisse être renversée par le commencement d'une preuve également fragile dont se prévalait le requérant. Mais c'était bien là le moins que pût le juge.

Pour ce qu'il est possible d'en savoir, le reste du contentieux de l'antisémitisme vichyssois - interdits professionnels, aryanisation économique, mesures de police, etc. - fut à l'avenant de cette rapide description de la jurisprudence de la qualification : toutes juridictions confondues, le froid juridisme s'y mêle à quelques exploits modestes, les assauts de résistance contre l'arbitraire du pouvoir à des solutions qui aggravèrent la rigueur des textes antijuifs eux-mêmes ${ }^{60}$.

24 À tenter d'expliquer certaines de ces décisions, par-delà l'évidence des contraintes politiques et institutionnelles plus ou moins fortes, historiens ou juristes contemporains ne peuvent faire l'économie de la psychologie judiciaire. Sans doute, les préjugés xénophobes et antisémites, couramment partagés avant-guerre dans la société française ${ }^{61}$ , constituent un facteur explicatif majeur de certaines errances. Assurément, l'accoutumance de l'ensemble de la communauté juridique - car dans ce contexte les avocats et les universitaires tinrent leur rôle aussi bien que les juges ${ }^{62}-$ à une révérence positiviste sans faille pour la loi, héritage de soixante-quinze ans de parlementarisme absolu, rend compte de nombreux aveuglements. Peut-être enfin ces derniers étaient-ils d'autant plus inévitables que les lois raciales de Vichy, actes de l'État français et non diktats de l'occupant, n'étaient pas loin de représenter, malgré tout, un motif de fierté pour un peuple en « souveraineté surveillée ».

En aval, il s'avère malaisé de connaître l'impact réel de ces interventions juridictionnelles durant la période, et notamment en quelle mesure elles ont pu influencer la pratique administrative quotidienne ${ }^{63}$. Il n'est en revanche guère douteux que cette activité de juges de droit commun, - quelle qu'en fût la nature et le sens : objectivement favorable ou défavorable aux Juifs, passif enregistrement de la norme antisémite, renforcement proprio 
motu de ses exigences ou, à l'inverse, habile subversion de ses effets recherchés -, recélait pour le régime la valeur d'une irremplaçable caution donnée à sa politique, pas seulement antisémite.

Sur un plan général, en reconnaissant le plein statut de loi aux actes ainsi dénommés par l'autocratie qui les édictait, et l'incontestabilité qui s'attachait au genre, les juridictions censeur de l'exécutif, le Conseil d'État d'abord - établissaient un continuum qui reliait la République à son contraire ; toute démocratie suspendue en l'absence d'assemblées représentatives, l'image perdurant de l'État de droit - le respect du Texte - suppléait sa réalité disparue. Ayant de la sorte admis ces dispositions dans le système juridique et auprès de ses techniciens, les juges les inscrivaient par suite - selon un processus que les travaux de Danièle Lochak notamment ont mis en évidence ${ }^{64}-$ comme également $^{4}$ recevables dans la sphère sociale et les mentalités du plus grand nombre. De ce point de vue, quant au droit antisémite, la normalisation de l'ignominie dans l'ordre juridique, banalisation du mal, concourant dans l'ordre symbolique à sa légitimation, il est certain qu'« en acceptant d'appliquer et d'interpréter les textes qui définissaient le "Juif", [les juges] ont contribué à faire admettre comme évidente l'idée que les juifs étaient d'une espèce - d'une " race "... - différente, qu'ils n'étaient pas des citoyens comme les autres, pas des sujets de droit comme les autres, et finalement pas des hommes comme les autres ${ }^{65} »$.

\section{BIBLIOGRAPHIE}

La plupart des contributions majeures intéressant le sujet ont été citées dans le cours de l'étude. Toute tentative d'exhaustivité étant illusoire en la matière, on se borne ci-dessous à quelques rappels.

Mission d'étude sur la spoliation des Juifs de France, La Persécution des Juifs de France, 1940-1944, et le rétablissement de la légalité républicaine, Recueil des textes officiels, 1940-1999, La Documentation française, 2000.

Il y a 50 ans : le statut des Juifs de Vichy, C.D.J.-C., 1991.

Le genre humain $\mathrm{n}^{\circ}$ 28, Juger sous Vichy, 1994.

Le genre humain $\mathrm{n}^{\circ}$ 30/31, Le droit antisémite de Vichy, 1996.

La Revue administrative, numéro spécial 1998, Le Conseil d'État et les crises.

BARUCH Marc Olivier, Servir l'État français : l'administration en

France de 1940 à 1944, Fayard, 1997.

FABRE Philippe, Le Conseil d'État et Vichy : le contentieux de l'antisémitisme, Publications de la Sorbonne, « De Rebus Publicis », 2000.

MARCoU Jean, Le Conseil d'État sous Vichy (1940-1944), thèse de doctorat (droit public), Grenoble II, 1984. 
MARRUS Michael, PAXTON Robert, Vichy et les Juifs, Calmann-Lévy, 1981 (rééd. Hachette, « Le Livre de poche $», 1990)$.

WEISBERG Richard, Vichy, la justice et les Juifs, Éditions des archives contemporaines (Amsterdam), 1998.

\section{NOTES}

1.On trouvera l'ensemble des textes antisémites cités dans un nouvel outil de travail : Mission d'étude sur la spoliation des Juifs de France, La persécution des Juifs de France, 1940-1944, et le rétablissement de la légalité républicaine, Recueil des textes officiels, 1940-1999, La Documentation française, 2000. (Les chercheurs ne disposaient jusque-là que de la recension réalisée en 1945 par le Centre de documentation juive, Les Juifs sous l'Occupation. Recueil des textes officiels français et allemands. 19401944, réédité en 1982 par l'Association Les Fils et Filles des Déportés Juifs de France).

2.V. d'une part R. Schor, L'Antisémitisme en France pendant les années trente. Prélude à Vichy, Complexe (Bruxelles), 1992 ; d'autre part M. Marrus et R. Paxton, Vichy et les Juifs, Calmann-Lévy, 1981 (rééd. Hachette, « Le Livre de poche », 1990), ch. 2, « Les origines de l'antisémitisme de Vichy ".

3.Cf. M. Marrus et R. Paxton, op. cit., et plus largement, du second, La France de Vichy. 1940-1944, Seuil, 1973 (1 $1^{\text {ère }}$ éd.), révolution dans l'historiographie de Vichy ( $2^{e}$ éd. 1997). V. note 13 ci-dessous.

4.P. Martin, « La haine, origine du droit », dans Pouvoir et liberté. Études offertes à Jacques Mourgeon, Bruylant (Bruxelles), 1998, p. 229-238 ; p. 238.

5.Ordonnance du 27 septembre 1940 : « Sont reconnus comme juifs ceux qui appartiennent ou appartenaient à la religion juive, ou qui ont plus de deux grandsparents (grands-pères et grands-mères) juifs. Sont considérés comme juifs les grandsparents qui appartiennent ou appartenaient à la religion juive ». Ce n'est qu'en avril 1941 que l'occupant reprendrait la seconde branche de la définition légale vichyssoise, « sorte de cas de « relaps » par le mariage » (J. Marcou, « La « qualité de Juif ». L'antisémitisme d'État, de la propagande au droit positif », Le Genre humain $n^{\circ} 30 / 31$, Le droit antisémite de Vichy, 1996, p. 153-172; p. 156).

6. V. J. Billig, Le Commissariat général aux questions juives (1941-1944), C.D.J.-C., 3 vol., 19551960 - aujourd'hui encore un travail de référence. V. aussi G. Simon, « L'administration de l'antisémitisme ", Le Genre humain n 30/31 précité, p. 307-325.

7.C'est par ce terme que Vallat se flatterait en 1947, devant la Haute Cour qui le jugerait, d'avoir su perfectionner le texte.

8.Article $1^{\mathrm{er}}$ du statut. V. L. Rosengart, «L'évolution de la définition raciale du Juif sous le régime de Vichy ", dans Il y a 50 ans : le statut des Juifs de Vichy, C.D.J.-C., 1991, p. 46-59. 9.V. J.-M. Béraud, « Le Juif interdit de travail », Le Genre humain n³0/31 précité, p. 189-208.

10.V. M. Blocaille-Boutelet, « L'« aryanisation » des biens », Le Genre humain n 30/31 précité, p. 243-266 ; Ph. Verheyde, Les Mauvais Comptes de Vichy. L'aryanisation des entreprises juives, Perrin, 1999.

11.Loi du 4 octobre 1940, publiée au Journal officiel en même temps que le premier statut des Juifs. V. A. Grynberg, Les Camps de la honte, La Découverte, 1991. La III ${ }^{\mathrm{e}}$ République 
finissante avait du reste largement anticipé ces internements, comme d'autres discriminations touchant les Juifs (v. R. Schor, op. cit.).

12.V. S. Klarsfeld, Vichy-Auschwitz : le rôle de Vichy dans la solution finale, Fayard, 2 vol. (t. I, 1940-1942 ; t. II, 1943), 1983-1985, et Le calendrier de la persécution des Juifs en France. 1940-1944, F.F.D.J.F., 1993. Pour une approche d'ensemble, outre l'ouvrage de référence précité de M. Marrus et R. Paxton, v. A. Kaspi, Les Juifs pendant l'occupation, Seuil, « Points », 1997 ( $2^{\mathrm{e}}$ éd.), et R. Poznanski, Les Juifs en France pendant la Seconde Guerre mondiale, Hachette, 1997 (2 e éd.).

13.V. d'une part J.-P. Azéma, «Vichy et la mémoire savante : quarante-cinq ans d'historiographie », in J.-P. Azéma et F. Bédarida (dir.), Le Régime de Vichy et les Français, Fayard, 1992, p. 23-44 ; d'autre part R. Poznanski, « Vichy et les Juifs. Des marges de l'histoire au cœur de son écriture ", ibid., p. 57-67.

14.Cf. H. Rousso, Le Syndrome de Vichy de 1940 à nos jours, Seuil, « Points », 1990 ( $2^{\mathrm{e}}$ éd. ; $1^{\text {ère }}$ éd. 1987), et, du même avec E. Conan, Vichy : un passé qui ne passe pas, Gallimard, « Folio »,

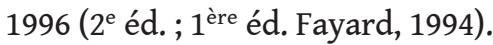

15.La question ne fut frontalement abordée, pour le principal, qu'à partir de deux colloques : en novembre 1993, sous les auspices de l'École nationale de la magistrature, Juger sous Vichy (Le Genre humain n² 28, 1994); en décembre 1995, à l'initiative de l'Université de Bourgogne, Le Droit antisémite de Vichy (Le genre humain n 30/31 précité). Dès 1991 toutefois, v. B. de Bigault du Granrut, «Les Tribunaux et le statut des Juifs », dans Il y a 50 ans, op. cit., p. 36-45.

16.Cf. D. Gros, « Un droit monstrueux ? », Le Genre humain n 30/31 précité, p. 561-575. Les normes antisémites, « perversion » du droit républicain dont elles bafouaient les valeurs tout en conservant ses mécanismes, n'en constituaient pas moins des règles juridiques à part entière. Mutatis mutandis, v. l'analyse du droit nazi qu'effectue D. de Béchillon, Qu'estce qu'une règle de droit ?, O. Jacob, 1997, p.255 sq.

17.V. Ch. Bachelier et D. Peschanski, « L'épuration de la magistrature sous Vichy », in Association française pour l'histoire de la justice, L'Épuration de la magistrature de la Révolution à la Libération, Loysel, 1994, p. 103-115 ; J.-P. Royer, Histoire de la justice en France, P.U.F., « Droit fondamental », 1996 (2 éd.), p. 713 sq.

18.Sur la problématique à l'œuvre, v. D. Lochak, « Le juge doit-il appliquer une loi inique ?", Le Genre humain n० 28, p. 29-39.

19.V. la synthèse dressée par P. Novick, L'épuration française. 1944-1949, Balland, 1985, rééd. Seuil, « Points », 1991, ann. : « La légitimité et la légalité de Vichy », p. 305 sq.

20.Lesquelles, avec les recueils officiels, constituent en la matière la seule source aisément accessible.

21.Juge administratif de droit commun, les conseils de préfecture ne connaissant encore - et jusqu'à la réforme de 1953 qui les transformerait en tribunaux administratifs qu'un contentieux d'attribution très spécialisé.

22.En témoignent ses deux premiers arrêts afférents au statut des Juifs, qui ne donnèrent pas lieu à l'évocation du problème, où le commissaire du gouvernement s'en tint à l'habituelle recherche de la volonté des auteurs du texte : Ass. 24 avril 1942 Lévy et BlocFavier, Rec. p. 135 ; G.P. 1942, II, jur., p. 192 ; J.-C.P. 1942, II, nº 1967 ; S. 1943, III, p. 25. « Qu'a voulu, en effet, le législateur? ", demandait le commissaire Léonard dans l'affaire Lévy, évoquant plus loin, pour s'en soucier encore, « le vœu du législateur » (cité par la Gazette du Palais, p. 194).

23.C.E. 22 mars 1944 Vincent, S. 1945, III, p. 53, note R.-E. Charlier - transposition discutable de Sect. 6 novembre 1936 Arrighi, S. 1937, III, concl. R. Latournerie, note A. 
Mestre, p. 33. Pour la discussion, v. O. Laederich, Étude juridique des épurations françaises de la Seconde Guerre mondiale. 1939-1953, thèse de doctorat (droit public), Paris II, 1999, p. 165 sq. et p. 519 sq.

24.Sur l'institution globalement appréhendée entre 1940 et 1944, v. L. Fougère (dir.), Le Conseil d'État, son histoire à travers les documents d'époque. 1799-1974, C.N.R.S., 1974, ch. XIII (19391945) ; J. Marcou, Le Conseil d'État sous Vichy (1940-1944), thèse de doctorat (droit public), Grenoble II, 1984 ; les deux contributions de J. Massot : « Le Conseil d'État », dans Le Régime de Vichy et les Français, op. cit., p. 312-328, et « Le Conseil d'État et le régime de Vichy ", Vingtième Siècle n 58, 1998, p. 83-99, repris in R.A., numéro spécial 1998, Le Conseil d'État et les crises, p. 46-52. V. aussi M. O. Baruch, Servir l'État français : l'administration en France de 1940 à 1944, Fayard, 1997, passim, et, du même, "Le Conseil d'État sous Vichy ", R.A., numéro spécial 1998 précité, p. 57-61.

25.V. P. Ancel, « La jurisprudence civile et commerciale », Le Genre humain $n^{\circ} 30 / 31$ précité, p. 363384.

26.Les revues spécialisées n'ont cependant conservé la trace que d'affaires relatives au recensement obligatoire. V. H. Bonnard, « La jurisprudence pénale », Le Genre humain n 30/31 précité, p. 385-396.

27.La distribution du contentieux de l'aryanisation, partagé de fait, durant toute la période, entre le Conseil d'État et les tribunaux civils, n'allait guère de soi. Les contorsions argumentatives auxquelles s'efforça alors la doctrine reflètent nettement cette difficulté à déterminer les principes qui auraient dû régir la question : v. par ex. E. Bertrand, « Du contrôle judiciaire du dessaisissement des juifs et de la liquidation de leurs biens (étude critique de jurisprudence) », J.-C.P. 1943, I, n 354. (Sur les juristes autres que juges sous Vichy, v. réf. note 62 ci-dessous.)

28.V. J.-P. Dubois, « La jurisprudence administrative », Le genre humain $n^{\circ}$ 30/31 précité, p. 339-362 ; v. aussi O. Dupeyroux, « L'indépendance du Conseil d'État statuant au contentieux », R.D.P. 1983, p. 565-629 (p. 608 sq.).

29.Parmi les premiers échos que les revues juridiques ont répercutés de ce contentieux civil de l'antisémitisme, v. par ex. Trib. civ. Seine 3 novembre 1941 Docteur Z. c/Veuve L., D. 1942, an., somm., p. 2 ; Trib. civ. Rabat, 17 décembre 1941 Lévy, J.-C.P. 1941, II, nº 1800 , rapport P. Decroux, obs. P. Chauveau.

30.Cf. Lévy et Bloc-Favier, précités note 22.

31.Note précitée de la Gazette du Palais sous l'arrêt Bloc-Favier, p. 193.

32.Terme employé par J. Marcou, op. cit., p. 239.

33.Ass. 2 avril 1943 Kaan, Rec. p. 85 ; G.P. 1943, I, jur., p. 176 ; S. 1944, III, note A. B., p. 6.

34.Note A. B. précitée.

35. Note de la Gazette du Palais sous l'arrêt Kaan, p. 177.

36.V. Trib. corr. Marseille, 25 février 1942 Weinthal, J.-C.P. 1942, II, nº 1823 ; C.A. Montpellier, 21 mai 1942 Lustac, G.P. 1942, II, jur., p. 6, et J.-C.P. 1942, nº 1968 ; C.A. Paris, 9 février 1943, G.P. 1943, I, jur., p. 103.

37. Comme le relève $\mathrm{H}$. Bonnard, loc. cit., p. 393. Le même note que seule une décision (Trib. corr. Toulouse, 22 décembre 1941 Dorfmann, D. 1942, cr., jur., note P. Chauveau, p. 53 ; J.-C.P. 1942, II, 1800 , obs. E.H. Perreau) « a commis cette erreur, mais dans une rédaction maladroite et avec la louable intention d'affirmer que l'infraction n'était pas constituée » : le jugement décida en effet qu' « attendu qu'il n'apparaît pas que Dorfmann soit juif au sens de la loi [...] l'élément fondamental [sic] du délit qui lui est reproché d'infraction à la loi sur le recensement des juifs fait donc défaut et [...] il doit être déclaré en voie de relaxe ». 
38.Du 7 janvier. D. 1944, an., jur., p. 34 ; G.P. 1944, I, jur., p. 41 ; J.-C.P. 1944, II, nº 2 566, obs. R. Savatier.

39.Les juges parisiens firent entre autre valoir, d'une part, que l'état des personnes qui justifie cette compétence « est constitué par l'ensemble des qualités inhérentes à la personne, que la loi prend en considération pour y attacher des effets juridiques ", et que " sont nécessairement au nombre des éléments constitutifs de cet état la race et la religion, à raison des effets juridiques que ces deux éléments sont susceptibles de produire » compte tenu des lois raciales; d'autre part, que « quelles que soient les difficultés d'interprétation qu'elles peuvent présenter, les questions de filiation auxquelles sont étroitement liées les questions de race, puisque la loi du 2 juin 1941 fait dépendre la qualité de juif ou d'aryen notamment de la race des grands-parents de l'intéressé, sont en toute hypothèse de la compétence des tribunaux civils », et qu' « il est donc sans intérêt de rechercher, ainsi que le propose le préfet de police, si une difficulté d'interprétation résulte ou non de l'application de la loi du 2 juin 1941 »-difficulté qui, en l'état du droit jurisprudentiel réglant la matière (T.C. 16 juin 1923 Septfonds), aurait imposé la saisine du juge administratif. Dans le même sens moins le détail de l'argumentation, v. Trib. civ. Sarlat, 3 mars 1944 Sarlat c/Galada, G.P. 1944, I, jur., p. 195. 40.V. par exemple le jugement cité note précédente (in fine) du Tribunal civil de Sarlat, qui dénie la judéité du défendeur « attendu que Gadala, conjoint d'une juive, n'est pas issu de deux grands-parents de race juive, étant entendu qu'est regardé comme de race juive le grand-parent ayant appartenu à la religion juive », et « qu'il n'a jamais appartenu à la confession juive » lui-même. V. a contrario Trib. civ. Rabat, 11 mars 1942 Griguer, J.-C.P. 1942, II, nº 1850 , obs. P. Chauveau - admettant la judéité de l'individu en cause en tant qu'il était issu de quatre grands-parents juifs, bien que ses parents et lui-même eussent adhéré à la religion catholique avant juin 1940, dans la mesure où une telle appartenance confessionnelle n'était prise en considération par le statut de 1941 qu'à l'égard des individus issus de deux grands-parents juifs seulement.

41.J. Marcou, op. cit., p. 246. Un exemple significatif est fourni par cet extrait d'un arrêt du Conseil d'État (Ass. 31 mars 1944 Dame Berthy, Rec. p. 107) : «Considérant qu'il est constant que la dame Berthy (Germaine-Rose), née Touati, est née à Alger le 15 février 1900 de Touati (Israël) et de Témine (Eugénie) ; que Touati (Israël), enfant naturel non reconnu, est né à Tlemsen le 25 avril 1857 et que son acte de naissance le désigne comme étant né de la Dame Semha Bant Saoud Touati ; que Témine (Eugénie), également enfant naturelle non reconnue, est née à Alger le 23 janvier 1874 et que son acte de naissance mentionne qu'elle est née de la Dame Témine Sarah... »

42.V. I. Lecoq-Caron, « La preuve de la qualité de Juif », Le Genre humain n² 28 précité, p. 41-52.

43.Pour un cas exprès, v. C.A. Montpellier, 17 décembre 1942 Avidor, G.P. 1943, I, jur., p. 55. Contra, cependant, v. le jugement Dorfmann précité (note 37) - mais c'était, là encore, pure maladresse.

44.Arrêt Hazan du 14 janvier 1943, D. 1943, cr., jur., rapport M. Nast, p. 33 ; G.P. 1943, I, jur., p. 57 ; J.-C.P. 1943, II, n 2 156, obs. M. Nast.

45.Arrêt du 12 mai, Demoiselle Weinthal, D. 1942, an., jur., p. 131 ; G.P. 1942, II, jur., p. 41 ; J.C.P. 1942, II, $\mathrm{n}^{\circ} 1$ 922, obs. P. Chauveau. V. de même Trib. corr. Bergerac, 12 juin 1942 Pierre Bloch, J.-C.P. 1941, II, n 1 940, obs. E.H. Perreau ; C.A. Paris, 26 octobre 1942 R.B., G.P. 1942, II, jur., p. 196, et S. 1943, III, p. 21 ; C.A. Montpellier, 17 décembre 1942 Avidor, G.P. 1943, I, jur., p. 56.

46.Précité, note 37. 
47.V. Trib. civ. Toulouse, 19 juin 1942 Dorfmann, G.P. 1942, II, jur., p. 51 ; J.-C.P. 1942, II, nº 1 931, obs. E.H. Perreau.

48.V. par ex. Trib. Civ. Bordeaux, 23 décembre 1942 Dame Foustet, G.P. 1943, I, jur., p. 68 reconnaissant la non-judéité de la femme qui, issue de deux grands-parents juifs, avait épousé un catholique et apportait notamment la preuve testimoniale que, athée, elle n'appartenait pas à la religion juive.

49.Jugement Lévy précité note 29. Cette interprétation, adoptée contre l'avis du rapporteur, fut censurée en appel : v. C.A. Rabat, 28 juillet 1942, J.-C.P. 1942, II, n 2 066, obs. E.H. Perreau.

50.Jugement du 15 janvier 1942, Benaïm, J.-C.P. 1942, II, n 1 825, obs. P. Chauveau. Un mariage effectué selon les formes canoniques, en revanche, authentifiait la réalité de l'appartenance à la religion catholique (4 février 1942, Achour, J.-C.P. 1942, II, n 1833 ). 51.Avis du 11 décembre 1942, G.P. 1943, I, jur., p. 295.

52.Arrêts Lévy et Kaan précités (notes 22 et 33) ; 13 juillet 1943 Dame veuve Molina, Rec. p. 186 ; 31 décembre 1943 Michelson, Rec. p. 311. Le raisonnement qui sous-tendait cette jurisprudence était l'œuvre du commissaire du gouvernement Léonard, concluant sur l'arrêt Lévy (V. G.P. 1942, II, p. 194).

53. C'est de cette façon qu'il admit qu'un ondoiement suffisait à faire entrer l'ondoyé dans la communauté des fidèles : v. l'arrêt Lévy précité (note 22) ; Sect. 30 avril 1943 Demoiselle Sée, Rec. p. 114, G.P. 1943, I, jur., p. 268, et S. 1943, III, p. 35 ; 17 décembre 1943 Sprecher, Rec. p. 294. On put toutefois faire observer à l'époque que le Conseil interprétait à sa manière le droit de l'Église romaine : v. la note anonyme au Sirey sur l'arrêt Lévy.

54.Ainsi de tel requérant qui ne produisait pas d'acte de baptême mais avait « dès l'année 1930 déclaré adhérer à la religion protestante ", s'était la même année " marié avec une protestante au temple », et avait " participé souvent depuis lors aux exercices du culte » (Sect. 5 février 1943 Alekan, Rec. p. 29) ; de même, de telle femme qui, bien qu'elle ne produisît pas non plus d'acte de baptême, se trouvait à l'identique mariée depuis longtemps à un protestant, et avait « élevé ses enfants suivant les principes de l'Église réformée ", tandis que « la paroisse protestante de Passy la regardait comme un de ses membres » (5 janvier 1944 Dame Touchon, Rec. p. 2).

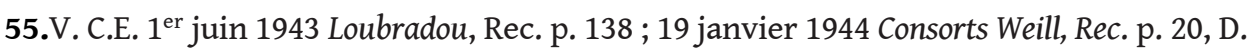
1944, an., jur., p. 65.

56.Rendu le même jour que l'arrêt Kaan précité (note 33), et commenté aux mêmes références.

57.V. à l'identique l'arrêt Rosengart rendu le 12 janvier 1944, Rec. p. 10.

58.Arrêt du 7 avril 1943, Willig, Rec. p. 89. À comparer avec l'arrêt Demoiselle Weinthal précité (réf. note 45$)$.

59.Michelson, précité note 52 .

60.Pour cette analyse, brevitatis causa, on ne peut que renvoyer à l'ouvrage précité, Le Conseil d'État et Vichy.

61.Cf. réf. note 2 ci-dessus.

62.V. d'une part R. Weisberg, « Les maîtres du barreau. Réflexions à partir des archives de Maurice Garçon ", Le Genre humain n³0/31, p. 401-412, repris dans l'ouvrage du même, Vichy, la justice et les Juifs, Éditions des archives contemporaines (Amsterdam), 1998, p. 94 sq. ; v. aussi R. Badinter, Un antisémitisme ordinaire. Vichy et les avocats juifs (1940-1944), Fayard, 1997. V. d'autre part B.-M. Bloch, «Le regard des juristes sur les lois raciales de Vichy ", Les Temps modernes n 547, 1992, p. 161-174 ; D. Gros, « Le statut des Juifs et les manuels en usage dans les facultés de droit sous le régime de Vichy ", dans Ph. Braud 
(dir.), La Violence politique dans les démocraties européennes occidentales, L'Harmattan, 1993, p. 139-171 ; D. Lochak, « La doctrine sous Vichy... », loc. cit. note 64 ci-dessous, et «Écrire, se taire... Réflexions sur l'attitude de la doctrine française ", Le Genre humain n 30/31, p. 433-462.

63.Il faudrait disposer de matériaux peu accessibles, concernant à l'échelon le plus élevé le C.G.Q.J. mais aussi, sur le plan local, ses agents déconcentrés et les services préfectoraux.

64.Cf.principalement ici « La doctrine sous Vichy ou les mésaventures du positivisme », dans C.U.R.A.P.P., Les Usages sociaux du droit, P.U.F., 1989, p. 252-285 ; v. aussi « Droit, normalité et normalisation ", dans C.U.R.A.P.P., Le Droit en procès, P.U.F., 1983.

65.D. Lochak, «Le juge doit-il appliquer une loi inique ?», loc. cit., p. 33.

\section{RÉSUMÉS}

À la définition de la judéité postulée par les statuts antisémites de Vichy les juges, comme à l'ensemble de l'application des dispositions anti-juives du régime, ont apporté une collaboration sans doute difficile à refuser, mais précieuse pour légitimer la politique ainsi conduite. S'il semble qu'en la matière les tribunaux répressifs surent à la fois éviter d'engager trop avant leur compétence et l'employer au mieux, les juridictions civiles et le Conseil d'État se partagèrent, non sans confusion, l'essentiel d'un contentieux de la qualification à l'origine d'une jurisprudence contrastée. Par un travail d'interprétation textuelle et de circonscription des moyens de preuve, tous contribuèrent à réifier cette abstraction idéologique millénaire : le « Juif ».

Building Identity

Jewish legal identity under Vichy regime : judges'contribution Judges collaborated to the definition of Jewish identity given by Vichy anti-semitic statutes, and to the whole application of the regime's anti-Jewish measures. This collaboration may have been difficult to refuse, but it has been precious to legitimate such a policy. The repressive courts seem to have managed to avoid involving their competence too far and to use it at best ; however, not without confusion, the civil juridictions and the State Council shared between themselves most of a litigation of the qualification leading to a contrasted jurisprudence. By a work of textual interpretation and by circonscribing the means of finding evidence, all of them contributed to freeze the millenial ideological abstraction which was juridically made objective between 1940 and 1944 : the « Jew».

\section{AUTEUR}

\section{PHILIPPE FABRE}

Philippe Fabre né en 1974, titulaire d'un DEA en droit public interne et diplômé de l'IEP de Paris, est actuellement allocataire-moniteur à l'Université Paris I - Panthéon-Sorbonne, où il prépare une thèse de doctorat sur L'Appréhension de la moralité par le droit public. ph.a.fabre(at)libertysurf.fr 